\title{
Location-based Mobile Augmented Reality Applications Challenges, Examples, Lessons Learned
}

\author{
Philip Geiger ${ }^{1}$, Marc Schickler ${ }^{1}$, Rüdiger Pryss ${ }^{1}$, Johannes Schobel ${ }^{1}$, Manfred Reichert $^{1}$ \\ ${ }^{1}$ Institute of Databases and Information Systems, University of Ulm, James-Franck-Ring, Ulm, Germany \\ \{philip.geiger, marc.schickler, ruediger.pryss, johannes.schobel, manfred.reichert $\} @$ uni-ulm.de
}

Keywords: $\quad$ Smart Mobile Applications, Location-based Mobile Augmented Reality.

\begin{abstract}
The technical capabilities of modern smart mobile devices more and more enable us to run desktop-like applications with demanding resource requirements in mobile environments. Along this trend, numerous concepts, techniques, and prototypes have been introduced, focusing on basic implementation issues of mobile applications. However, only little work exists that deals with the design and implementation (i.e., the engineering) of advanced smart mobile applications and reports on the lessons learned in this context. In this paper, we give profound insights into the design and implementation of such an advanced mobile application, which enables location-based mobile augmented reality on two different mobile operating systems (i.e., iOS and Android). In particular, this kind of mobile application is characterized by high resource demands since various sensors must be queried at run time and numerous virtual objects may have to be drawn in realtime on the screen of the smart mobile device (i.e., a high frame count per second be caused). We focus on the efficient implementation of a robust mobile augmented reality engine, which provides location-based functionality, as well as the implementation of mobile business applications based on this engine. In the latter context, we also discuss the lessons learned when implementing mobile business applications with our mobile augmented reality engine.
\end{abstract}

\section{INTRODUCTION}

Daily business routines increasingly require access to information systems in a mobile manner, while requiring a desktop-like feeling of mobile applications at the same time. However, the design and implementation of mobile business applications constitutes a challenging task (Robecke et al., 2011). On one hand, developers must cope with limited physical resources of smart mobile devices (e.g., limited battery capacity or limited screen size) as well as non-predictable user behaviour (e.g., mindless instant shutdowns). On the other, mobile devices provide advanced technical capabilities, including motion sensors, a GPS sensor, and a powerful camera system. Hence, new types of business applications can be designed and implemented in the large scale. Integrating sensors and utilizing the data recorded by them, however, is a non-trivial task when considering requirements like robustness and scalability as well. Moreover, mobile business applications have to be developed for different mobile operating systems (e.g., iOS and Android) in order to allow for their widespread use. Hence, developers of mobile business applications must also cope with the heterogeneity of exist- ing mobile operating systems, while at the same time utilizing their technical capabilities. In particular, if mobile application users shall be provided with the same functionality in the context of different mobile operating systems, new challenges may emerge when considering scalability and robustness. This paper deals with the development of a generic mobile application, which enables location-based mobile augmented reality for realizing advanced business applications. We discuss the core challenges emerging in this context and report on the lessons learned when applying it to implement real-world mobile business applications. Existing related work has been dealing with location-based mobile augmented reality as well (Fröhlich et al., 2006; Carmigniani et al., 2011; Paucher and Turk, 2010; Reitmayr and Schmalstieg, 2003). To the best of our knowledge, they do not focus on aspects regarding the efficient integration of location-based mobile augmented reality with realworld mobile business applications.

\subsection{PROBLEM STATEMENT}

The overall purpose of this work is to show how to develop the core of a location-based mobile augmented 
reality engine for the mobile operating systems $i O S$ 5.1 (or higher) and Android 4.0 (or higher). We denote this engine as $A R E A^{1}$. As a particular challenge, the augmented reality engine shall be able to display points of interest (POIs) from the surrounding of a user on the screen of his smart mobile device. In particular, POIs shall be drawn based on the angle of view and the position of the smart mobile device. This means that the real image captured by the camera of the smart mobile device will be augmented by virtual objects (i.e., the POIs) relative to the current position and attitude. The overall goal is to draw POIs on the camera view of the smart mobile device.

The development of a mobile augmented reality engine constitutes a non-trivial task. In particular, the following challenges emerge:

- In order to enrich the image captured by the smart mobile device's camera with virtual information about POIs in the surrounding, basic concepts enabling location-based calculations need to be developed.

- An efficient and reliable technique for calculating the distance between two positions is required (e.g., based on data of the GPS sensor in the context of outdoor location-based scenarios).

- Various sensors of the smart mobile device must be queried correctly in order to determine the attitude and position of the smart mobile device.

- The angle of view of the smart mobile device's camera lens must be calculated to display the virtual objects on the respective position of the camera view.

Furthermore, a location-based mobile augmented reality engine should be provided for all established mobile operating systems. However, to realize the same robustness and ease-of-use for heterogenous mobile operating systems, is a non-trivial task.

\subsection{CONTRIBUTION}

In the context of AREA, we developed various concepts for coping with the limited resources on a smart mobile device, while realizing advanced features with respect to mobile augmented reality at the same time. In this paper, we present a sophisticated application architecture, which allows integrating augmented reality with a wide range of applications. However, this architecture must not neglect the characteristics of the

\footnotetext{
${ }^{1}$ AREA stands for Augmented Reality Engine Application. A video demonstrating AREA can be viewed at: http://vimeo.com/channels/434999/63655894. Further information can be found at: http://www.area-project.info
}

underlying kind of mobile operating system. While in many scenarios the differences between mobile operating systems are rather uncrucial when implementing a mobile business application, for the present mobile application this does no longer apply. Note that there already exist augmented reality frameworks and applications for mobile operating systems like Android or iOS. These include proprietary and commercial engines as well as open source frameworks and applications (Lee et al., 2009; Wikitude, 2013). To the best of our knowledge, however, these proposals neither provide insights into the functionality of such an engine nor its customization to a specific purpose. Furthermore, insights regarding the development of engines running on more than one mobile operating systems are usually not provided. To remedy this situation, we report on the lessons learned when developing AREA and integrating it with our mobile business applications.

This paper is organized as follows: Section 2 introduces core concepts and the architecture of AREA. In Section 3, we discuss lessons learned when implementating AREA on the iOS and Android mobile operating systems. In particular, this section discusses differences we experienced in this context. Section 4 gives detailed insights into the use of AREA for implementing real-world business applications. In Section 5 related work is discussed. Section 6 concludes the paper with a summary and outlook.

\section{AREA APPROACH}

The basic concept realized in AREA is the locationView. The points of interest inside the camera's field of view are displayed on it, having a size of $\sqrt{\text { widt }^{2}+\text { height }^{2}}$ pixels. The locationView is placed centrally on the screen of the mobile device.

\subsection{The locationView}

Choosing the particular approach provided by the $l o$ cationView has specific reasons, which we discuss in the following.

First, AREA shall display points of interest (POIs) correctly, even if the device is hold obliquely. Depending on the device's attitude, the POIs then have to be rotated with a certain angle and moved relatively to the rotation. Instead of rotating and moving every POI separately in this context, however, it is also possible to only rotate the locationView to the desired angle, whereas the POIs it contains are rotated automatically; i.e., resources needed for complex calculations can be significantly reduced. 

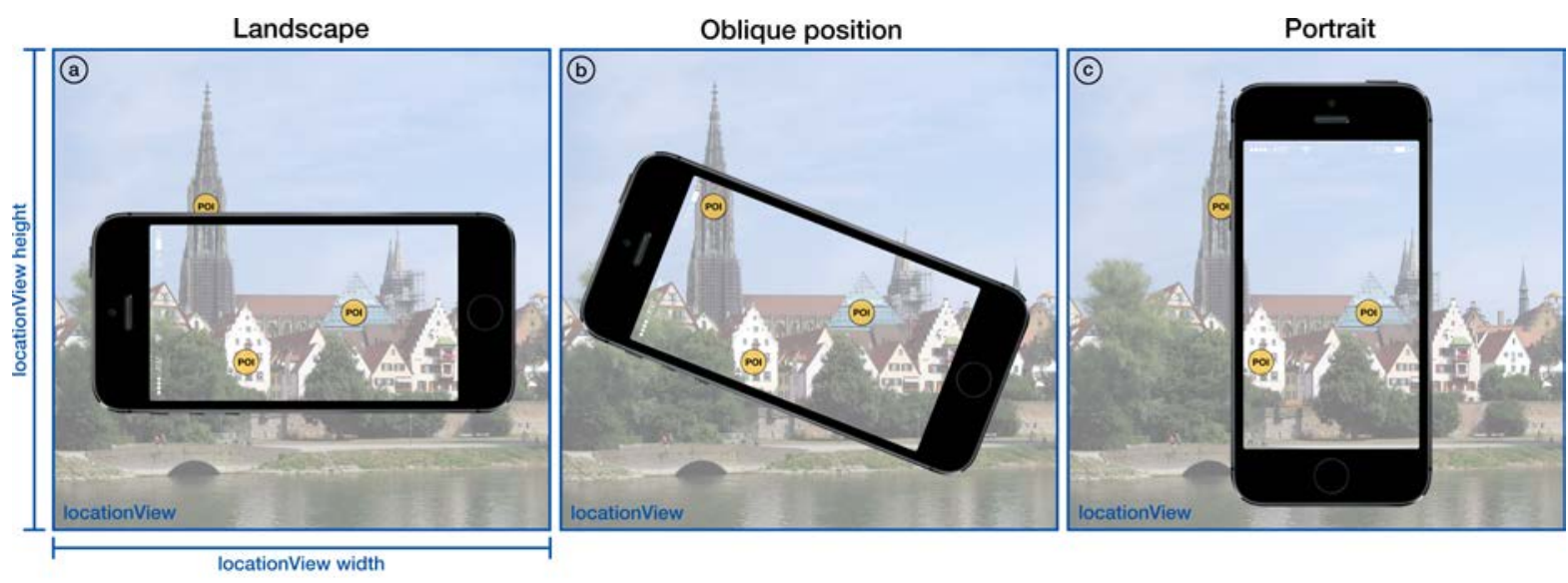

Figure 1: Examples of locationView depicting its characteristics.

Second, a complex recalculation of the field of view of the camera is not required if the device is in an oblique position. The vertical and horizontal dimensions of the field of view are scaled proportionally to the diagonal of the screen, such that a new maximum field of view results with the size of $\sqrt{w_{i d t h}+h e i g h t^{2}}$ pixels. Since the locationView is placed centrally on the screen, the camera's actual field of view is not distorted. Further, it can be customized by rotating it contrary to the rotation of the device. The calculated maximal field of view is needed to efficiently draw POIs visible in portrait mode, landscape mode, or any oblique position inbetween.

Fig. 1 presents an example illustrating the concept of the locationView. Thereby, each sub-figure represents one locationView. As one can see, a locationView is bigger than the display of the respective mobile device. Therefore, the camera's field of view must be increased by a certain factor such that all POIs, which are either visible in portrait mode (cf. Fig. 1c), landscape mode (cf. Fig. 1a), or any rotation inbetween (cf. Fig. 1b), are drawn on the locationView. For example, Fig. 1a shows a POI (on the top) drawn on the locationView, but not yet visible on the screen of the device in landscape mode. Note that this POI is not visible for the user until he rotates his device to the position depicted in Fig. 1b. Furthermore, when rotating the device from the position depicted in Fig. 1b to portrait mode (cf. Fig. 1c), the POI on the left disappears again from the field of view, but still remains on the locationView.

The third reason for using the presented locationView concept concerns performance. When the display has to be redrawn, the POIs already drawn on the locationView can be easily queried and reused. Instead of first clearing the entire screen and after- wards re-initializing and redrawing already visible POIs, POIs that shall remain visible, do not have to be redrawn. Furthermore, POIs located outside the field of view after a rotation are deleted from it, whereas POIs that emerge inside the field of view are initialized.

Fig. 2 sketches the basic algorithm used for realizing this locationView ${ }^{2}$.

\subsection{Architecture}

The AREA architecture has been designed with the goal to be able to easily exchange and extend its components. The design comprises four main modules organized in a multi-tier architecture and complying with the Model View Controller pattern (cf. Fig. 3). Lower tiers offer their services and functions by interfaces to upper tiers. In particular, the red tier (cf. Fig. 3) will be described in detail in Section 3, when discussing the differences regarding the development of AREA on the iOS and Android platforms. Based on this architectural design, modularity can be ensured; i.e., both data management and various elements (e.g., the POIs) can be customized and extended on demand. Furthermore, the compact design of AREA enables us to build new mobile business applications based on it as well as to easily integrate it with existing applications.

The lowest tier, called Model, provides modules and functions to exchange the POIs. In this context, we use both an $X M L$ - and a $J S O N$-based interface to collect and parse POIs. In turn, these POIs are then stored in a global database. Note that we do not rely on the ARML schema (ARML, 2013), but use our own

\footnotetext{
${ }^{2}$ More technical details can be found in a technical report (Geiger et al., 2013)
} 


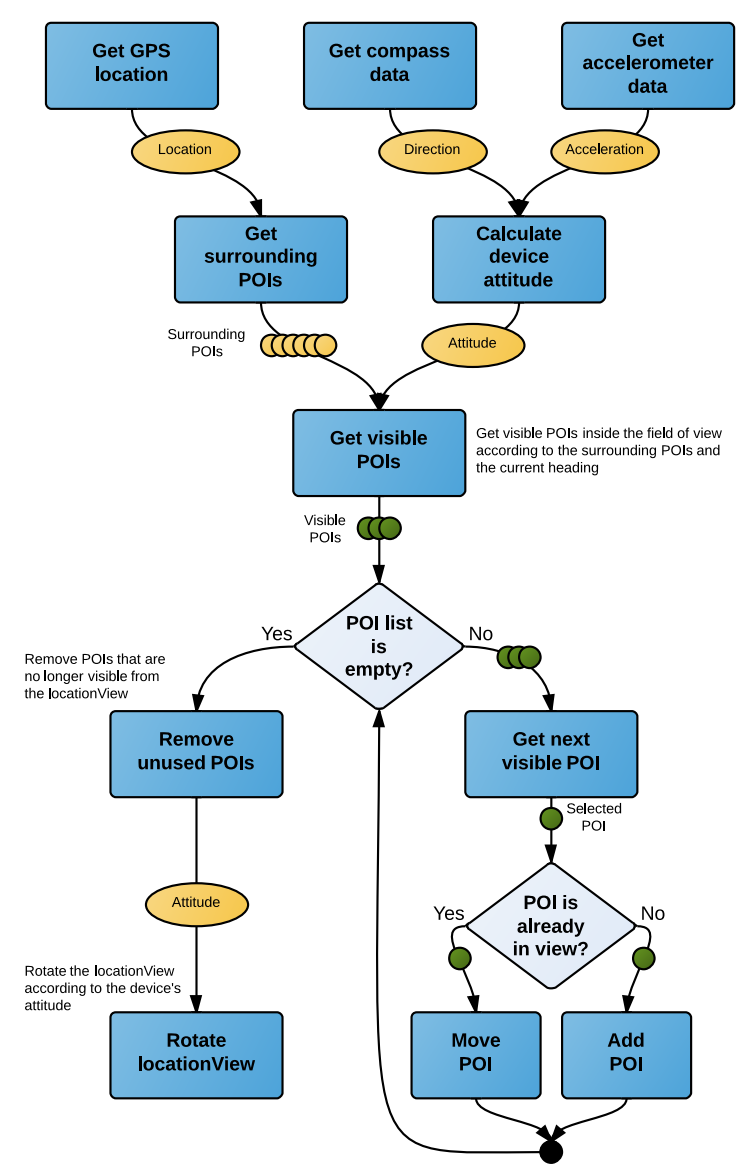

Figure 2: Algorithm realizing the locationView (sketched).

XML schema. In particular, we will be able to extend our $X M L$-based format in the context of future research on AREA. Finally, the JSON interface uses a light-weight, easy to understand, and extendable format with which developers are familiar.

The next tier, called Controller, consists of two main modules. The Sensor Controller is responsible for culling the sensors necessary to determine the device's location and orientation. The sensors to be culled include the GPS sensor, the accelerometer, and the compass sensor. The GPS sensor is used to determine the position of the device. Since we currently focus on location-based outdoor scenarios, GPS coordinates are predominantly used. In future work, we address indoor scenarios as well. Note that the architecture of AREA has been designed to easily change the way coordinates will be obtained. Using the GPS coordinates and its corresponding altitude, we can calculate the distance between mobile device and POI, the horizontal bearing, and the vertical bearing. The latter is used to display a POI higher or lower on the screen, depending on its own altitude. In turn, the accelerometer provides data for determining the current rotation of the device, i.e., the orientation of the device (landscape, portrait, or any other orientation inbetween) (cf. Fig. 1). Since the accelerometer is used to determine the vertical viewing direction, we need the compass data of the mobile device to determine the horizontal viewing direction of the user as well. Based on the vertical and horizontal viewing directions, we are able to calculate the direction of the field of view as well as its boundaries according to the camera angle of view of the device. The Point of Interest Controller (cf. Fig. 3) uses data of the Sensor Controller in order to determine whether a POI is inside the vertical and horizontal field of view. Furthermore, for each POI it calculates its position on the screen taking the current field of view and the camera angle of view into account.

The uppermost tier, called View, consists of various user interface elements, e.g., the locationView, the Camera View, and the specific view of a POI (i.e., the Point of Interest View). Thereby, the Camera View displays the data captured by the device's camera. Right on top of the Camera View, the locationView is placed. It displays POIs located inside the current field of view at their specific positions as calculated by the Point of Interest Controller. To rotate the locationView, the interface of the Sensor Controller is used. The latter allows to determining the orientation of the device. Furthermore, a radar can be used to indicate the direction in which invisible POIs are located (cf. Fig. 9 shows an example of the radar). Finally, AREA make use of libraries of the mobile development frameworks themselves, which provide access to core functionality of the underlying operating system, e.g., sensor access and screen drawing functions (cf. Native Frameworks in Fig. 3).

\section{EXPERIENCES WITH IMPLEMENTING AREA ON EXISTING MOBILE OPERATING SYSTEMS}

The kind of business application we consider utilizes the various sensors of smart mobile devices, and hence provides new kinds of features compared to traditional business applications. However, this significantly increases complexity for application developers as well. This complexity further increases if the mobile application shall be provided for different mobile operating systems.

Picking up the scenario of mobile augmented reality, this section gives insights into ways for effi- 


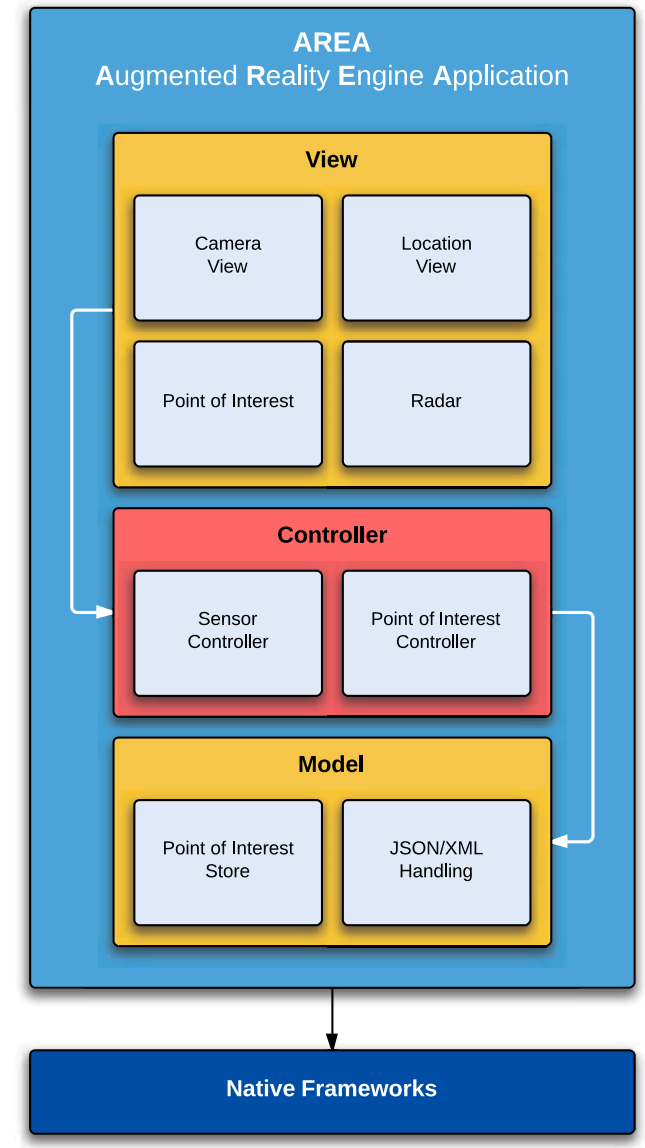

Figure 3: Multi-tier architecture of AREA.

ciently handling the POIs, relevant for the locationView of our mobile augmented reality engine. In this context, the implementation of the Sensor Controller and the Point of Interest Controller are most interesting regarding the subtle differences one must consider when developing such an engine on different mobile operating systems (i.e., iOS and Android).

In order to reach a high efficiency when displaying or redrawing POIs on the screen, we choose a native implementation of AREA on the iOS and Android mobile operating systems. Thus, we can make use of provided built-in APIs of these operating systems, and can call native functions without any translation as required in frameworks like Phonegap (Systems, 2013). Note that efficiency is very crucial for mobile business applications (Corral et al., 2012) since smart mobile devices rely on battery power. Therefore, to avoid high battery usage by expensive framework translations, only a native implementation is appropriate in our context. Apart from this, most crossplatform development frameworks do not provide a proper set of functions to work with sensors (Schobel et al., 2013). In the following, we present the implementation of AREA on both the iOS and the Android mobile operating systems.

\section{1 iOS MOBILE OPERATING SYSTEM}

The iOS version of AREA has been implemented using the programming language Objective-C and iOS Version 7.0 on Apple iPhone 4S. Furthermore, for developing AREA, the Xcode environment (Version 5) has been used.

\subsubsection{Sensor Controller}

The Sensor Controller is responsible for culling the necessary sensors in order to correctly position the POIs on the screen of the smart mobile device. To achieve this, iOS provides the CoreMotion and CoreLocation frameworks. We use the CoreLocation framework to get notified about changes of the location as well as compass heading. Since we want to be informed about every change of the compass heading, we adjusted the heading filter of the CoreLocation framework accordingly. When the framework sends us new heading data, its data structure contains a real heading as well as a magnetic one as floats. The real heading complies to the geographic north pole, whereas the magnetic heading refers to the magnetic north pole. Since our coordinates corresponds to GPS coordinates, we use the real heading data structure. Note that the values of the heading will become (very) inaccurate and oscillate when the device is moved. To cope with this, we apply a lowpass filter (Kamenetsky, 2013) to the heading in order to obtain smooth and accurate values, which can then be used to position the POIs on the screen. Similar to the heading, we can adjust how often we want to be informed about location changes. On one hand, we want to get notified about all relevant location changes; on the other, every change requires a recalculation of the surrounding POIs. Thus, we deciced to get notified only if a difference of at least 10 meters occurs between the old and the new location. Note that this is generally acceptable for the kind of applications we consider (cf. Section 4.1). Finally, the data structure representing a location contains GPS coordinates of the device in degrees north and degrees east as decimal values, the altitude in meters, and a time stamp.

In turn, the CoreMotion framework provides interfaces to cull the accelerometer. The accelerometer is used to calculate the current rotation of the device as well as to determine in which direction the smart mobile device is pointing (e.g., in upwards or down- 
wards direction). As opposed to location and heading data, accelerometer data is not automatically pushed by the iOS CoreMotion framework to the application. Therefore, we had to define an application loop that is polling this data every $\frac{1}{90}$ seconds. On one hand, this rate is fast enough to obtain smooth values; on the other, it is low enough to save battery power. As illustrated by Fig. 4, the data the accelerometer delivers consists of three values, i.e., the accelerations in $\mathrm{x}-, \mathrm{y}-$, and $\mathrm{z}$-direction ((Apple, 2013)). Since gravity is required for calculating in which direction a device is pointing, but we cannot obtain this gravity directly using the acceleration data, we had to additionally apply a lowpass filter (Kamenetsky, 2013), i.e., the filter is used for being applied to the $\mathrm{x}-, \mathrm{y}-$, and $\mathrm{z}$-direction values. Thereby, the three values obtained are averaged and filtered. In order to obtain the vertical heading as well as the rotation of the device, we then have to apply the following steps: First, by calculating $\arcsin (z)$, we obtain a value between $\pm 90^{\circ}$ and describing the vertical heading. Second, by calculating $\arctan 2(-y, x)$, we obtain a value between $0^{\circ}$ and $359^{\circ}$, describing the degree of the amount of the rotation of the (Alasdair, 2011) of the device.

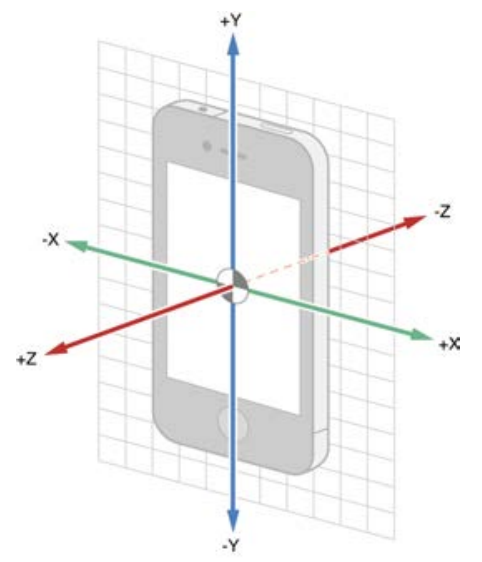

Figure 4: The three axes of the iPhone acceleration sensor (Apple, 2013).

Since we need to consider all possible orientations of the smart mobile device, we must adjust the compass data accordingly. For example, assume that we hold the device in portrait mode in front of us towards the North. Then, the compass data we obtain indicate that we are viewing in northern direction. As soon as we rotate the device, however, the compass data will change, although our view still goes to northern direction. This is caused by the fact that the reference point of the compass corresponds to the upper end of the device. To cope with this issue, we must adjust the compass data using the above presented rotation calculation. When subtracting the rotation value (i.e., $0^{\circ}$ and $359^{\circ}$ ) from the compass data, we obtain the desired compass value, still viewing in northern direction after rotating the device (cf. Fig. 5).

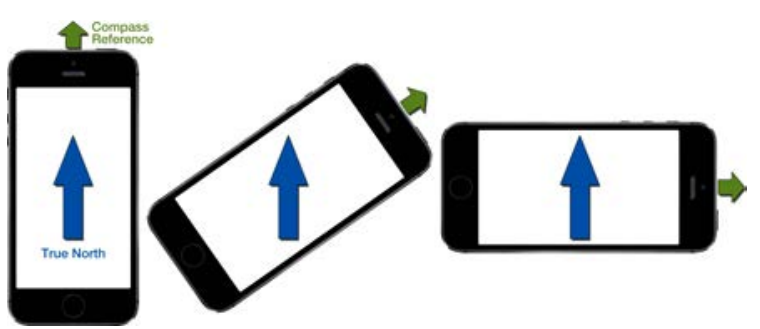

Figure 5: Adjusting the compass data to the device's current rotation.

\subsubsection{Point of Interest Controller}

As soon as the Sensor Controller has collected the required data, it notifies the Point of Interest Controller at two points in time: (1) when detecting a new location and (2) after having gathered new heading as well as accelerometer data. When a new location is detected, we must determine the POIs in the surrounding of the user. For this purpose, we use an adjustable radius (see Fig. 9 for an example of such an adjustable radius). By using the latter, a user can determine the maximum distance she has to the POIs to be displayed. By calculating the distance between the device and the POIs based on their GPS coordinates (Bullock, 2007), we can determine the POIs located inside the chosen radius and hence the POIs to be displayed on the screen. Since only POIs inside the field of view (i.e., POIs actually visible for the user) shall be displayed on the screen, we must further calculate the vertical and horizontal bearing of the POIs inside the radius. Due to space limitation, we cannot describe these calculations in detail, but refer interested readers to a technical report (Geiger et al., 2013). As explained in this report, the vertical bearing can be calculated based on the altitudes of the POIs and the smart mobile device (the latter can be determined from the current GPS coordinates). In turn, the horizontal bearing can be computed using the Haversine formula (Sinnott, 1984) and applying it to the GPS coordinates of the POI and the smart mobile device. Finally, in order to avoid recalculations of these surrounding POIs in case the GPS coordinates do not change (i.e., within movings of $10 \mathrm{~m}$ ), we must buffer data of the POIs inside the controller implementation for efficiency reasons.

As a next step, the heading and accelerometer data need to be processed when obtaining a notification from the Sensor Controller (i.e., the application loop mentioned in Section 3.1.1 has delivered new data). 
Based on this, we can determine whether or not a POI is located inside the vertical and horizontal field of view, and at which position it shall be displayed on the locationView. Recall that the locationView extends the actual field of view to a larger, orientationindependent field of view (cf. Fig. 6). The first step is to determine the boundaries of the locationView based on sensor data. In this context, the heading data provides the information required to determine the direction the device is pointing at. The left boundary of the locationView can be calculated by determining the horizontal heading and decreasing it by the half of the maximal angle of view (cf. Fig. 6). The right boundary is calculated by adding half of the maximal angle of view to the current heading. Since POIs have also a vertical heading, a vertical field of view must be calculated as well. This is done analogously to the calculation of the horizontal field of view, except that the data of the vertical heading is required. Finally, we obtain a directed, orientation-independent field of view bounded by left, right, top, and bottom values. Then we use the vertical and horizontal bearings of a POI to determine whether it lies inside the locationView (i.e., inside the field of view). Since we use the concept of the locationView, we do not have to deal with the rotation of the device at this point, i.e., we can normalize calculations to portrait mode since the rotation itself is handled by the locationView.

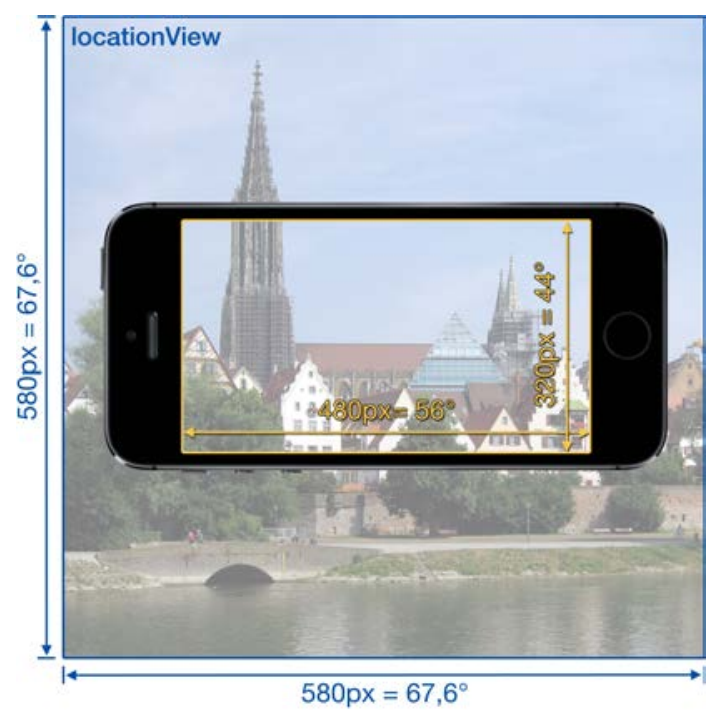

Figure 6: Illustration of the new maximal angle view and the real one.

The camera view can be created and displayed applying the native AVFoundation framework. Using the screen size of the device, which can be determined at run time, the locationView can be initialized and placed centrally on top of the camera view. As soon as the Point of Interest Controller has finished its calculations (i.e., it has determined the positions of the POIs), it notifies the View Controller that organizes the view components. The View Controller then receives the POIs and places them on the locationView. Recall that in case of a device rotation, only the locationView must be rotated. As a consequence, the actual visible field of view changes accordingly. Therefore, the Point of Interest Controller sends the rotation of the device calculated by the Sensor Controller to the View Controller, together with the POIs. Thus, we can adjust the field of view by simply counterrotating the locationView using the given angle. Based on this, the user will only see those POIs on his screen, being inside the actual field of view. In turn, other POIs will be hidden after the rotation, i.e., moved out of the screen (cf. Fig. 1). Detailed insights into respective implementation issues, together with well described code samples, can be found in (Geiger et al., 2013).

\subsection{ANDROID MOBILE OPERATING SYSTEM}

In general, mobile business applications should be made available on all established platforms in order to reach a large number of users. Hence, we developed AREA for the Android mobile operating system as well (and will also make it available for Windows Phone at a later point in time). This section gives insights into the Android implementation of AREA, comparing it with the corresponding iOS implementation. Although the basic software architecture of AREA is the same for both mobile operating systems, there are differences regarding its implementation.

\subsubsection{Sensor Controller}

For implementing the Sensor Controller, the packages android.location and android.hardware are used. The location package provides functions to retrieve the current GPS coordinate and altitude of the respective device, and is similar to the corresponding $\mathrm{iOS}$ package. However, the Android location package additionally allows retrieving an approximate position of the device based on network triangulation. Particularly, if no GPS signal is available, the latter approach can be applied. However, as a drawback, no information about the current altitude of the device can be determined in this case. In turn, the hardware package provides functions to get notified about the current magnetic field and accelerometer. The latter corresponds to the one of iOS, and is used to calculate the rotation of the device. However, the heading is calculated in a different way compared to iOS. In- 
stead of obtaining it with the location service, it must be determined manually. Generally, the heading depends on the rotation of the device and the magnetic field. Therefore, we create a rotation matrix using the data of the magnetic field (i.e., a vector with three dimensions) and the rotation based on the accelerometer data. Since the heading data depends on the accelerometer as well as the magnetic field, it is rather inaccurate. More precisely, the calculated heading is strongly oscillating. Hence, we apply a lowpass filter to mitigate this oscillation. Note that this lowpass filter is of another type than the one used in Section 3.1.1 for calculating the gravity.

Moreover, as soon as other magnetic devices are located nearby the actual mobile device, the heading will be distorted. In order to notify the user about the presence of such a disturbed magnetic field, leading to false heading values, we apply functions of the hardware package. Another difference between iOS and Android concerns the way the required data can be obtained. Regarding iOS, location-based data is pushed, whereas sensor data must be polled. As opposed to iOS, on Android all data is pushed by the framework, i.e., application programmers rely on Android internal loops and trust the up-to-dateness of the data provided. Note that such subtle differences between mobile operating systems and their development frameworks should be well understood by the developers of advanced mobile business applications.

\subsubsection{Point of Interest Controller}

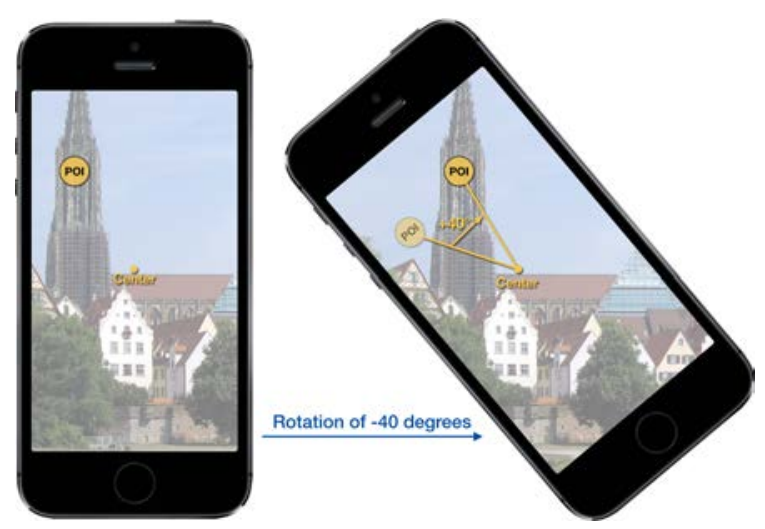

Figure 7: Android specific rotation of POI and field of view.

Regarding Android, the Point of Interest Controller works the same way as the one of iOS. However, when developing AREA we had to deal with one particular issue. The locationView manages the visible POIs as described above. Therefore, it must be able to add child views (e.g., every POI generating one child view). As described in Section 3.1, on iOS
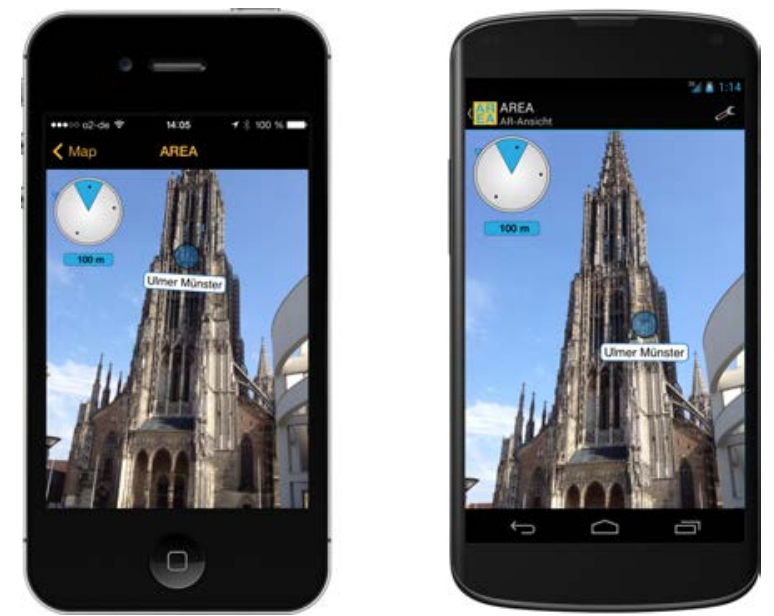

Figure 8: AREA's user interface for iOS and Android.

we simply rotate the locationView to actually rotate the POIs and the field of view. In turn, on Android, a layout containing child views cannot be rotated in the same way. Thus, when the Point of Interest Controller receives sensor data from the Sensor Controller, the $\mathrm{x}$ - and $\mathrm{y}$-coordinates of the POIs must be determined in a different way. Instead of placing the POIs independently of the current rotation of the device, we make use of the degree of rotation provided by the Sensor Controller. Following this, the POIs are rotated around the centre of the locationView and we also rotate the POIs about their centres (cf. Fig. 7). Using this approach, we can still add all POIs to the field of view of the locationView. Finally, when rotating the POIs, they will automatically leave the device's actual field of view.

\subsection{COMPARISON}

This section compares the two implementations of AREA on iOS and Android. First of all, it is noteworthy that the features and functions of the two implementations are the same. Moreover, the user interfaces realized for AREA on iOS and Android, respectively, are the same (see Fig. 8).

\subsubsection{Realizing the locationView}

The developed locationView with its specific features differs between the Android and iOS implementations of AREA. Regarding the iOS implementation, we could realize the locationView concept as described in Section 2.1. On the Android operating system, however, not all features of this concept worked properly. More precisely, extending the actual field of view of the device to the bigger size of the locationView worked well. Furthermore, determining whether 
or not a POI is inside the field of view, independent of the rotation of the device, worked also well. By contrast, rotating the locationView with its POIs to adjust the visible field of view as well as moving invisible POIs out of the screen did not work as easy on Android as expected. As particular problem in the given context, a simple view on Android must not contain any child views. Therefore, on Android we had to use the layout concept for realizing the described locationView. However, simply rotating a layout does not work on all Android devices. For example, on a Nexus 4 device this worked well by implementing the algorithm in exactly the same way as on iOS. In turn, on a Nexus 5 device this led to failures regarding the redraw process. When rotating the layout on the Nexus 5, the locationView is clipped by the camera surface view, which is located behind our locationView. As a consequence, to ensure that AREA is compatible with a wider set of Android devices, running Android 4.0 or later, we made the adjustments described in Section 4.2.

\subsubsection{Accessing Sensors}

Using sensors on the two mobile operating systems is different as well. The latter concerns the access to the sensors as well as their preciseness and reliability. Regarding iOS, the location sensor is offering the GPS coordinates as well as the compass heading. This data is pushed to the application by the underlying service offered by iOS. Concerning Android, the location sensor only provides data of the current location. Furthermore, this data must be polled by the application. The heading data, in turn, is calculated by the fusion of several motion sensors, including the accelerometer and magnetometer. The accelerometer is used on both platforms to determine the current orientation of the device. However, the preciseness of data provided differs significantly. Running and compiling the AREA engine on iOS with iOS 6 results in very reliable compass data with an interval of one degree. Running and compiling the AREA engine with iOS 7, however, leads to different results compared to iOS 6 . As advantage, iOS 7 enables a higher resolution of the data intervals provided by the framework due to the use of floating point data instead of integers. In turn, the partial unreliability of the delivered compass data is disadvantageous. Regarding iOS 7, compass data started to oscillate within an interval when moving the device. Therefore, we needed to apply a stronger lowpass filter in order to compensate this oscillating data. In turn, on Android the internal magnetometer, which is necessary for calculating the heading, is vulnerable to noisy sources (e.g., other devices, magnets, or computers). Thus, it might happen that the delivered data is unreliable and the application must wait until more reliable sensor data becomes available.

Furthermore, for each sensor the corresponding documentation on the respective operating system should be studied in detail in order to operate with them efficiently. In particular, the high number of different devices running Android constitutes a challenge when deploying AREA on the various hardware and software configurations of manufacturers. Finally, we learned that Android devices are often affected by distortions of other electronic hardware and, therefore, the delivered data might be unreliable as well.

Overall, the described differences demonstrate that developing advanced mobile business applications, which make use of the technical capabilities of modern smart mobile devices, is far from being trivial from the viewpoint of application developers.

\section{VALIDATION}

This section deals with the development of business applications with AREA and the lessons learned in this context.

\subsection{DEVELOPING BUSINESS APPLICATIONS WITH AREA}

AREA has been integrated with several business applications. For example, one company uses AREA for its application LiveGuide (CMCityMedia, 2013). A LiveGuide can be used to provide residents and tourists of a German city with the opportunity to explore their surrounding by displaying points of interests stored for that city (e.g., public buildings, parks, places of events, or companies). When realizing such business applications on top of AREA, it turned out that their implementation benefits from the modular design and extensibility of AREA. Furthermore, an efficient implementation could be realized. In particular, when developing the LiveGuide application type, only the following two steps were required: First, the appearance of the POIs was adapted to meet the user interface requirements of the respective customers. Second, the data model of AREA was adapted to an already existing one. On the left side of Fig. 9, we show user interface elements we made in the context of the LiveGuide applications. In turn, on the right side of Fig. 9, we show the user interface elements originally implemented for AREA. 

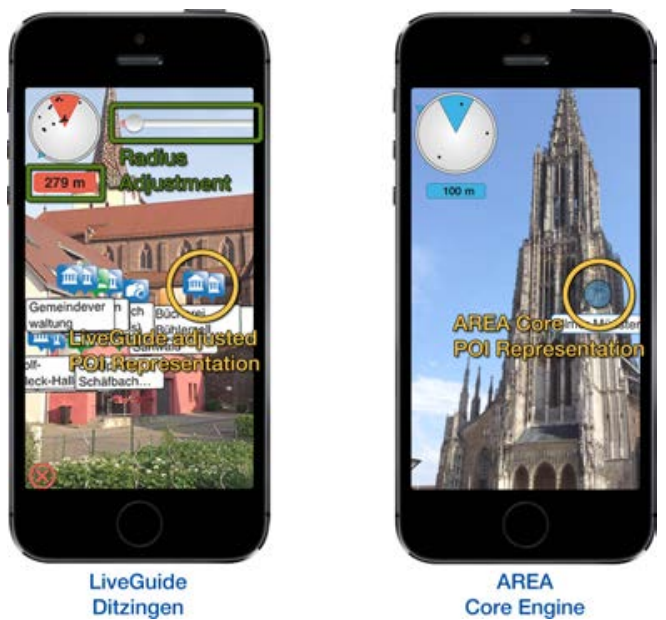

Figure 9: Typical adapted user interface provided by a LiveGuide application.

\subsection{LESSONS LEARNED}

This section discusses issues that emerged when developing business applications (e.g., LiveGuide) on top of AREA. Note that we got many other practical insights from the use of AREA. However, to set a focus, we restrict ourselves to two selected issues.

\subsubsection{Updates of Mobile Operating Systems}

As known, the iOS and Android mobile operating systems are frequently updated. In turn, respective updates must be carefully considered when developing and deploying an advanced mobile business application like AREA. Since the latter depends on the availability of accurate sensor data, fundamental changes of the respective native libraries might affect the proper execution of AREA. As example, consider the following issue we had to cope with in the context of an update of the Android operating system (i.e., the switch from Android Version 4.2 to Version 4.3). In the old version, the sensor framework notifies AREA when measured data becomes unreliable. However, with the new version of the mobile operating system, certain constants (e.g., SENSOR_STATUS_UNRELIABLE) we had used were no longer known on respective devices (cf. Fig. 10). To deal with this issue, the respective constant had to be replaced by a listener (cf. Fig. 10 onAccuracyChanged). As another example consider the release of iOS 7, which led to a change of the look and feel of the entire user interface. In particular, some of the customized user interface elements in the deployed version of the LiveGuide applications got hidden from one moment to the other or did not react to user interactions anymore. Thus, the application had to be

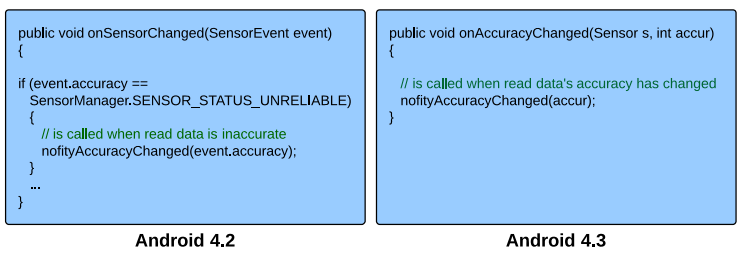

Figure 10: SENSOR_STATUS_UNRELIABLE change in Android 4.3.

fixed. Altogether, we learned that adjusting mobile applications due to operating system updates might cause considerable efforts.

\subsubsection{POI Data Format}

Using our own proprietary XML schema instead of applying and adapting the open source schema ARML has its pros and cons. On one hand, we can simply extend and modify this schema, e.g., to address upcoming issues in future work. On the other, when integrating AREA with the LiveGuide application, we also revealed drawbacks of our approach. In particular, the data format of POIs, stored in external databases, differs due to the use of a non-standardized format. Thus, the idea of ARML (ARML, 2013) is promising. Using such a standardized format for representing POIs from different sources should be pursued. Therefore, we will adapt AREA to support this standard with the goal to allow for an easy integration of AREA with other business applications.

\section{RELATED WORK}

Previous research related to the development of a location-based augmented reality application, which is based on GPS coordinates and sensors running on head-mounted displays, is described in (Feiner et al., 1997) and (Kooper and MacIntyre, 2003). In turn, a simple smart mobile device, extended by additional sensors, has been applied by (Kähäri and Murphy, 2006) to develop an augmented reality system. Another application using augmented reality is described in (Lee et al., 2009). Its purpose is to share media data and other information in a real-world environment and to allow users to interact with this data through augmented reality. However, none of these approaches addresses location-based augmented reality on smart mobile devices as AREA does. In particular, these approaches do not give insights into the development of such business applications.

The increasing size of the smart mobile device market as well as the technical maturity of smart mobile devices has motivated software vendors to 
realize augmented reality software development kits (SDKs). Example of such SDKs included Wikitude (Wikitude, 2013), Layar (Layar, 2013), and Junaio (Junaio, 2013). Besides these SDKs, there are popular applications like Yelp (Yelp, 2013), which use additional features of augmented reality to assist users when interacting with their surrounding.

Only little work can be found, which deals with the development of augmented reality systems in general. As an exception, (Grubert et al., 2011) validates existing augmented reality browsers. However, neither commercial software vendors nor scientific results related to augmented reality provide any insight into how to develop a location-based mobile augmented reality engine.

\section{SUMMARY \& OUTLOOK}

The purpose of this paper was to give insights into the development of the core framework of an augmented reality engine for smart mobile devices. We have further shown how business applications can be implemented based on the functionality of this mobile engine. As demonstrated along selected implementation issues, such a development is very challenging. First of all, a basic knowledge about mathematical calculations is required, i.e., formulas to calculate the distance and heading of points of interest on a sphere in the context of outdoor scenarios. Furthermore, deep knowledge about the various sensors of the smart mobile device is required from application developers, particularly regarding the way the data provided by these sensors can be accessed and processed. Another important issue concerns resource and energy consumption. Since smart mobile devices have limited resources and performance capabilities, the points of interest should be displayed in an efficient way and without delay. Therefore, the calculations required to handle sensor data and to realize the general screen drawing that must be implemented as efficient as possible. The latter has been accomplished through the concept of the locationView, which allows increasing the field of view and reusing already drawn points of interest. In particular, the increased size allows the AREA engine to easily determine whether or not a point of view is inside the locationView without considering the current rotation of the smart mobile device. In addition, all displayed points of interest can be rotated easily.

We argue that an augmented reality engine like AREA must provide a sufficient degree of modularity to enable a full and easy integration with existing applications as well as to implement new applications on top of it. Finally, it is crucial to realize a proper architecture and class design, not neglecting the communication between the components. We have further demonstrated how to integrate AREA in a realworld business applications (i.e., LiveGuide) and how to make use of AREA's functionality. In this context, the respective application has been made available in the Apple App and Android Google Play Stores. In particular, the realized application has shown high robustness. Finally, we have given insights into the differences between Apple's and Google's mobile operating systems when developing AREA.

Future research on AREA will address the challenges we identified during the implementation of the LiveGuide business application. For example, in certain scenarios the POIs located in the same direction overlap each other, making it difficult for users to precisely touch POIs. To deal with this issue, we are working on algorithms for detecting clusters of POIs and offering a way for users to interact with these clusters. In (Feineis, 2013), a component for on-thetrail navigation in mountainous regions has been developed on top of AREA, which is subject of current research as well. Furthermore, we are developing a marker-based augmented reality component in order to integrate marker based with location based augmented reality. Since GPS is only available for outdoor location, but AREA should also for indoor scenarios, we are working towards this direction as well. In the latter context, we use Wi-Fi triangulation to determine the device's indoor position (Bachmeier, 2013). Second, we are experiencing with the iBeacons approach introduced by Apple.

Finally, research on business process management offers flexible concepts, which are useful for enabling proper exception handling in the context of mobile applications as well (Pryss et al., 2012; Pryss et al., 2013; Pryss et al., 2010). Since mobile augmented reality applications may cause various errors (e.g., sensor data is missing), adopting these concepts is promising.

\section{REFERENCES}

Alasdair, A. (2011). Basic Sensors in iOS: Programming the Accelerometer, Gyroscope, and More. O'Reilly Media.

Apple (2013). Event handling guide for iOS: Motion events. [Online; accessed 10.12.2013].

ARML (2013). Augmented reality markup language. http://openarml.org/wikitude4. html. [Online; accessed 10.12.2013]. 
Bachmeier, A. (2013). Wi-fi based indoor navigation in the context of mobile services. Master Thesis, University of Ulm.

Bullock, R. (2007). Great circle distances and bearings between two locations. [Online; accessed 10.12.2013].

Carmigniani, J., Furht, B., Anisetti, M., Ceravolo, P., Damiani, E., and Ivkovic, M. (2011). Augmented reality technologies, systems and applications. Multimedia Tools and Applications, 51(1):341-377.

CMCityMedia (2013). City liveguide. http:// liveguide.de. [Online; accessed 10.12.2013].

Corral, L., Sillitti, A., and Succi, G. (2012). Mobile multiplatform development: An experiment for performance analysis. Procedia Computer Science, 10(0):736 - 743 .

Feineis, L. (2013). Development of an augmented reality component for on the trail navigation in mountainous regions. Master Thesis, University of Ulm, Germany.

Feiner, S., MacIntyre, B., Höllerer, T., and Webster, A. (1997). A touring machine: Prototyping 3d mobile augmented reality systems for exploring the urban environment. Personal Technologies, 1(4):208-217.

Fröhlich, P., Simon, R., Baillie, L., and Anegg, H. (2006). Comparing conceptual designs for mobile access to geo-spatial information. Proc of the 8th Conf on Human-computer Interaction with Mobile Devices and Services, pages 109112.

Geiger, P., Pryss, R., Schickler, M., and Reichert, M. (2013). Engineering an advanced location-based augmented reality engine for smart mobile devices. Technical Report UIB-2013-09, University of Ulm, Germany.

Grubert, J., Langlotz, T., and Grasset, R. (2011). Augmented reality browser survey. Technical report, Institute for Computer Graphics and Vision, Graz University of Technology, Austria.

Junaio (2013). Junaio. http: //Www . junaio. com/. [Online; accessed 11.06.2013].

Kähäri, M. and Murphy, D. (2006). Mara: Sensor based augmented reality system for mobile imaging device. 5th IEEE and ACM Int'l Symposium on Mixed and Augmented Reality.

Kamenetsky, M. (2013). Filtered audio demo. http://www. stanford.edu/ boyd/ ee102/conv_demo.pdf. [Online; accessed 17.01.2013].

Kooper, R. and MacIntyre, B. (2003). Browsing the real-world wide web: Maintaining awareness of virtual information in an AR information space. Int'l Journal of Human-Computer Interaction, $16(3): 425-446$.

Layar (2013). Layar. http://www. layar.com/. [Online; accessed 11.06.2013].

Lee, R., Kitayama, D., Kwon, Y., and Sumiya, K. (2009). Interoperable augmented web browsing for exploring virtual media in real space. Proc of the 2nd Int'l Workshop on Location and the Web, page 7 .

Paucher, R. and Turk, M. (2010). Location-based augmented reality on mobile phones. IEEE Computer Society Conf on Computer Vision and Pattern Recognition Workshops (CVPRW), pages 916.

Pryss, R., Langer, D., Reichert, M., and Hallerbach, A. (2012). Mobile task management for medical ward rounds - the MEDo approach. Proc BPM'12 Workshops, 132:43-54.

Pryss, R., Musiol, S., and Reichert, M. (2013). Collaboration support through mobile processes and entailment constraints. Proc 9th IEEE Int'l Conf on Collaborative Computing (CollaborateCom'13).

Pryss, R., Tiedeken, J., Kreher, U., and Reichert, M. (2010). Towards flexible process support on mobile devices. Proc CAiSE'10 Forum - Information Systems Evolution, (72):150-165.

Reitmayr, G. and Schmalstieg, D. (2003). Location based applications for mobile augmented reality. Proc of the Fourth Australasian user interface conference on User interfaces, pages 65-73.

Robecke, A., Pryss, R., and Reichert, M. (2011). Dbischolar: An iphone application for performing citation analyses. Proc CAiSE'11 Forum at the 23rd Int'l Conf on Advanced Information Systems Engineering, (Vol-73).

Schobel, J., Schickler, M., Pryss, R., Nienhaus, H., and Reichert, M. (2013). Using vital sensors in mobile healthcare business applications: Challenges, examples, lessons learned. Int'l Conf on Web Information Systems and Technologies, pages 509-518.

Sinnott, R. (1984). Virtues of the haversine. Sky and telescope, 68:2:158.

Systems, A. (2013). Phonegap. http://phonegap. com. [Online; accessed 10.12.2013].

Wikitude (2013). Wikitude. http://www. wikitude.com. [Online; accessed 11.06.2013].

Yelp (2013). Yelp. http: / / www . yelp.com. [Online; accessed 11.06.2013]. 